\title{
Two Point Correlation Functions for a Periodic Box-Ball System ${ }^{\star}$
}

\author{
Jun $M A D A^{\dagger}$ and Tetsuji TOKIHIRO ${ }^{\ddagger}$ \\ $\dagger$ College of Industrial Technology, Nihon University, \\ 2-11-1 Shin-ei, Narashino, Chiba 275-8576, Japan \\ E-mail: mada.jun@nihon-u.ac.jp \\ $\ddagger$ Graduate School of Mathematical Sciences, University of Tokyo, \\ 3-8-1 Komaba, Tokyo 153-8914, Japan \\ E-mail:toki@ms.u-tokyo.ac.jp
}

Received December 13, 2010, in final form March 02, 2011; Published online March 21, 2011 doi:10.3842/SIGMA.2011.027

\begin{abstract}
We investigate correlation functions in a periodic box-ball system. For the second and the third nearest neighbor correlation functions, we give explicit formulae obtained by combinatorial methods. A recursion formula for a specific $N$-point functions is also presented.
\end{abstract}

Key words: correlation function; box-ball system

2010 Mathematics Subject Classification: 37B15; 37K10; 81R12; 82B20

\section{Introduction}

A periodic box-ball system (PBBS) is a soliton cellular automaton obtained by ultradiscretizing the $\mathrm{KdV}$ equation $[1,2]$. It can also be obtained at the $q \rightarrow 0$ limit of an integrable lattice model (a generalised 6 vertex model) $[3,4]$. Let $V_{1}$ be a 2-dimensional complex vector space with basis $\vec{e}_{0}$ and $\vec{e}_{1}$. If we consider $N$ tensor product space of $V_{1}, V:=V_{1}^{\otimes N}$, the transfer matrix of the generalised 6 vertex model is a map (endmorphism) $T_{k}(x ; q): V \rightarrow V$ with a spectral parameter $x$ and a deformation parameter $q$. The positive integer $k$ denotes that the dimension of the auxiliary vector space is $k+1$. An important property of the transfer matrices is their commutativity: $T_{k}(u ; q) T_{l}(v ; q)=T_{l}(v ; q) T_{k}(u ; q)$ for arbitrary $k, l, u, v$. Hence they consist a complete set of diagonal operators and the lattice models are integrable. It is also noted that the Hamiltonian of the quantum $X X Z$ spin model is essentially given by $H_{X X Z}:=\lim _{x \rightarrow 1} \frac{\partial}{\partial x} \log T_{1}(x ; q)$. If we take a limit $\lim _{q \rightarrow 0, x \rightarrow 1} T(x ; q)=: T$, the transfer matrix $T$ maps each monomial $\left|i_{1} i_{2} \cdots i_{N}\right\rangle:=\vec{e}_{i_{1}} \otimes \vec{e}_{i_{2}} \otimes \cdots \otimes \vec{e}_{i_{n}} \in V$ to a monomial. By identifying $\left|i_{1} i_{2} \cdots i_{N}\right\rangle$ with a 10 sequence $i_{1} i_{2} \cdots i_{N}, T$ gives the time evolution of the PBBS. Using this relation, we can obtain several important properties of the PBBS such as the conserved quantities, a relation to the string hypothesis, a fundamental cycle and so on.

One of the main problems of quantum integrable systems now is to obtain correlation functions which is fairly difficult even for the $X X Z$ model and the 6 vertex model [6]. Since a correlation function of the $X X Z$ model or the 6 vertex model is, roughly speaking, transformed to a correlation function of the PBBS in the limit $q \rightarrow 0$. For example, a two point function $\left\langle s_{i}^{z} s_{j}^{z}\right\rangle$ of the $X X Z$ spin model is transformed to the probability that both $i$ th and

\footnotetext{
*This paper is a contribution to the Proceedings of the Conference "Integrable Systems and Geometry" (August 12-17, 2010, Pondicherry University, Puducherry, India). The full collection is available at http://www.emis.de/journals/SIGMA/ISG2010.html
} 


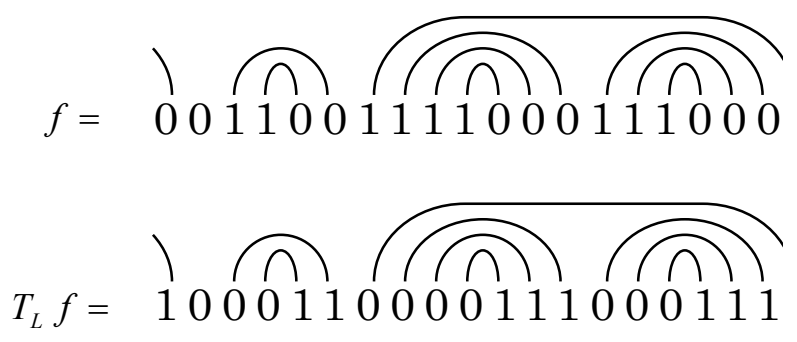

Figure 1. Definition of $T_{L}$ for $f \in \Omega_{L}$.

$j$ th componets of the PBBS are 1 . Hence, from the view point of integrable lattice models or quantum integrable models, it may actually give some new insights into the correlation functions of the vertex models themselves to obtain correlation functions of the PBBS.

In [5], we gave expressions for $N$-point functions using the solution for the PBBS expressed in terms of the ultradiscrete theta functions. We also gave expressions for the one point, the nearest and the second nearest neighbor correlation functions. Note that the correlation functions defined there and in this article are not the direct $q \rightarrow 0$ limit of the correlation functions of the corresponding lattice model; such a limit generally gives trivial results [5]. We define the correlation functions on the phase space of a set of fixed conserved quantities. In this article, we show that the second nearest neighbor correlation function can be simplified with some combinatorial formulae, and give an explicit formula for the third nearest neighbor correlation function by combinatorial methods. A recursion formula for a specific $N$-point function is also presented.

The PBBS can be defined in the following way. Let $L \geq 3$ and let $\Omega_{L}=\{f \mid f:[L] \rightarrow$ $\{0,1\}$ such that $\left.\sharp f^{-1}(\{1\})<L / 2\right\}$ where $[L]=\{1,2, \ldots, L\}$. When $f \in \Omega_{L}$ is represented as a sequence of $0 \mathrm{~s}$ and $1 \mathrm{~s}$, we write

$$
f(1) f(2) \cdots f(L) .
$$

The mapping $T_{L}: \Omega_{L} \rightarrow \Omega_{L}$ is defined as follows (see Fig. 1):

1. In the sequence $f$ find a pair of positions $n$ and $n+1$ such that $f(n)=1$ and $f(n+1)=0$, and mark them; repeat the same procedure until all such pairs are marked. Note that we always use the convention that the position $n$ is defined in $[L]$, i.e. $n+L \equiv n$.

2. Skipping the marked positions we get a subsequence of $f$; for this subsequence repeat the same process of marking positions, so that we get another marked subsequence.

3. Repeat part 2 until one obtains a subsequence consisting only of 0s. A typical situation is depicted in Fig. 1. After these preparatory processes, change all values at the marked positions simultaneously; One thus obtains the sequence $T_{L} f$.

The pair $\left(\Omega_{L}, T_{L}\right)$ is called a PBBS of length $L[2,7]$. An element of $\Omega_{L}$ is called a state, and the mapping $T_{L}$ the time evolution.

The conserved quantities of the PBBS are defined as follows. Let $Q_{j}(f)$ be the number of 10 pairs in $f$ marked at $j$ th step in the definition of the mapping $T_{L}$. Then we obtain a nonincreasing sequence of positive integers, $Q_{j}(f)(j=1,2, \ldots, m)$. This sequence is conserved in time, that is,

$$
Q_{j}(f)=Q_{j}\left(T_{L} f\right) \equiv Q_{j} \quad(j=1,2, \ldots, m) .
$$

For example, $\left(Q_{1}, Q_{2}, Q_{3}, Q_{4}\right)=(3,3,2,1)$ for $f$ given in Fig. 1 . As the sequence $\left(Q_{1}, Q_{2}, \ldots, Q_{m}\right)$ is nonincreasing, we can associate a Young diagram with it by regarding $Q_{j}$ as the number of 
squares in the $j$ th column of the diagram. The lengths of the rows are also weakly decreasing positive integers. Let the distinct row lengths be $P_{1}>P_{2}>\cdots>P_{\ell}$ and let $n_{j}$ be the number of times that the length $P_{j}$ appears. The set $\left\{P_{j}, n_{j}\right\}_{j=1}^{\ell}$ is another expression for the conserved quantities of the PBBS.

First we summarize some useful properties of the PBBS. We say that $f$ has (or that there is) a 10-wall at position $n$ if $f(n)=1$ and $f(n+1)=0$. Let the number of the 10-walls be $s$ and the positions be denoted by $a_{1}<a_{2}<\cdots<a_{s}$.

We introduce a procedure called 10-insertion. For $\left(b_{1}, b_{2}, \ldots, b_{L}\right) \in \mathbb{Z}_{\geq 0} \times \mathbb{Z}_{\geq 0} \times \cdots \times \mathbb{Z}_{\geq 0}(\equiv$ $\left.\left(\mathbb{Z}_{\geq 0}\right)^{L}\right)$ and $d:=\sum_{i=1}^{L} b_{i}$, the 10 -insertion is defined as $I\left(b_{1}, b_{2}, \ldots, b_{L}\right):=I_{2}\left(b_{1}, b_{2}, \ldots, b_{L}\right) \circ I_{1}$, where $I_{1}: \Omega_{L} \rightarrow \Omega_{L+2 s}$ is the mapping:

$$
\left(I_{1} f\right)(n)= \begin{cases}1 & \left(n=a_{j}+2 j-1, j \in[s]\right), \\ 0 & \left(n=a_{j}+2 j, j \in[s]\right), \\ f(n) & \left(1 \leq n \leq a_{1}\right), \\ f(n-2 j) & \left(a_{j}+2 j+1 \leq n \leq a_{j+1}+2 j, j \in[s-1]\right), \\ f(n-2 s) & \left(a_{s}+2 s+1 \leq n \leq L+2 s\right)\end{cases}
$$

and the mapping $I_{2}\left(b_{1}, b_{2}, \ldots, b_{L}\right): \Omega_{L+2 s} \rightarrow \Omega_{L+2(d+s)}$ is defined to be

$$
\left(I_{2}\left(b_{1}, b_{2}, \ldots, b_{L}\right) f^{\prime}\right)(n)= \begin{cases}f^{\prime}\left(n-2 \hat{b}_{j-1}\right) & \left(n=j+2 \hat{b}_{j-1}, j \in[L]\right), \\ 1 & \left(n=j+2 \hat{b}_{j-1}+2 h-1, j \in[L], h \in\left[b_{j}\right]\right), \\ 0 & \left(n=j+2 \hat{b}_{j-1}+2 h, j \in[L], h \in\left[b_{j}\right]\right),\end{cases}
$$

where $f^{\prime} \equiv I_{1} f \in \Omega_{L+2 s}, b_{0}=0$ and $\hat{b}_{j}:=\sharp\left\{i \in[s] \mid a_{i} \leq j\right\}+\sum_{i=1}^{j} b_{i}$. For example,

$$
\begin{array}{ll}
f & =0011100111000001101000111000000, \\
I_{1} f & =00111 \underline{10} 00111[100000011 \underline{10} 01[10000111 \underline{10} 000000, \\
\left.I_{(1}, 1_{22}, 1_{29}\right) f & =00111 \underline{10} 0011 \underline{101} 100000011[1001[1000010111100000 \underline{10} 00,
\end{array}
$$

where $\left(1_{9}, 1_{22}, 1_{29}\right)$ denotes the 10 sequence whose 9,22 , and 29 th elements are 1 and the others are 0 , that is,

$$
\left(1_{9}, 1_{22}, 1_{29}\right) \equiv(0,0,0,0,0,0,0,0,1,0,0,0,0,0,0,0,0,0,0,0,0,1,0,0,0,0,1,0,0,0,0),
$$

and the expressions 10 and $\underline{10}$ denote the 10s inserted at $f \mapsto I_{1} f$ and at $I_{1} f \mapsto I_{2}\left(b_{1}, b_{2}, \ldots\right.$, $\left.b_{L}\right)\left(I_{1} f\right)$ respectively.

\section{Correlation functions of PBBS}

We consider the PBBS with $L$ boxes and $M$ balls. As shown in [5], the correlation functions are defined on the states with the same conserved quantities. Let $Y$ be the Young diagram corresponding to the partition of $M$ :

$$
(\underbrace{P_{1}, P_{1}, \ldots, P_{1}}_{n_{1}}, \underbrace{P_{2}, P_{2}, \ldots, P_{2}}_{n_{2}}, \ldots, \underbrace{P_{\ell}, P_{\ell}, \ldots, P_{\ell}}_{n_{\ell}})
$$

where $P_{1}>P_{2}>\cdots>P_{\ell} \geq 1$ and $\sum_{j=1}^{\ell} n_{j} P_{j}=M$. The number of squares in the $j$ th largest raw of $Y$ is $P_{j}$. In the PBBS, the conserved quantities are characterized by the Young diagram $Y$ [8]. 
When we denote by $\Omega_{Y}$ the set of states with conserved quantities given by $Y$, the $N$-point function of the PBBS is defined as

$$
\left\langle s_{1}, s_{2}, \ldots, s_{N}\right\rangle_{Y}=\frac{1}{\left|\Omega_{Y}\right|} \sum_{f \in \Omega_{Y}} f\left(s_{1}\right) f\left(s_{2}\right) \cdots f\left(s_{N}\right) .
$$

Since the $N$-point function $\left\langle s_{1}, s_{1}+d_{1}, \ldots, s_{1}+d_{N-1}\right\rangle_{Y}$ does not depend on the specific site $s_{1}$ (because of translational symmetry), we denote

$$
C_{Y}\left(d_{1}, d_{2}, \ldots, d_{N-1}\right) \equiv\left\langle s_{1}, s_{1}+d_{1}, \ldots, s_{1}+d_{N-1}\right\rangle_{Y}
$$

where $1 \leq d_{1}<d_{2}<\cdots<d_{N-1}<L$. The 1-point function $C_{Y}(\varnothing):=\left\langle s_{1}\right\rangle_{Y}$ and the 2-point function $C_{Y}(1):=\left\langle s_{1}, s_{1}+1\right\rangle_{Y}$ are easily calculated as $C_{Y}(\varnothing)=\frac{M}{L}$ and $C_{Y}(1)=\frac{M-s}{L}$ where $s=\sum_{i=1}^{\ell} n_{i}$

Although general $N$-point functions are difficult to evaluate by elementary combinatorial methods, the correlation functions $C_{Y}(1,2, \ldots, N-1)=\left\langle s_{1}, s_{1}+1, s_{1}+2, \ldots, s_{1}+N-1\right\rangle_{Y}$ enjoy a simple recursion formula. Let us put

$$
k_{i}:=\left\{\begin{array}{ll}
n_{j} & \left(i=P_{j}\right), \\
0 & \text { otherwise },
\end{array} \quad \hat{k}_{i}:=\sum_{j=i}^{P_{1}} k_{j}, \quad L_{0}:=L, \quad L_{i}:=L_{i-1}-2 \hat{k}_{i},\right.
$$

for $i=1,2, \ldots, P_{1}$. Note that $k_{i}$ and $\hat{k}_{i}$ are the number of the rows in $Y$ with the length $i$ and that with the length greater than or equal to $i$ respectively. For the Young diagram $Y$, let $Y_{i}$ be the Young diagram corresponding to the partition

$$
\begin{gathered}
(\underbrace{\max \left[P_{1}-i, 0\right], \max \left[P_{1}-i, 0\right], \ldots, \max \left[P_{1}-i, 0\right]}_{n_{1}}, \\
\underbrace{\max \left[P_{2}-i, 0\right], \max \left[P_{2}-i, 0\right], \ldots, \max \left[P_{2}-i, 0\right]}_{n_{2}}, \ldots \\
\ldots \underbrace{\max \left[P_{\ell}-i, 0\right], \max \left[P_{\ell}-i, 0\right], \ldots, \max \left[P_{\ell}-i, 0\right]}_{n_{\ell}}) .
\end{gathered}
$$

The following two lemmas immediately follow from the definition of $I_{1}$ and the procedure of 10-insertion.

Lemma 1. In a state $f=I_{1} f_{1}\left(f_{1} \in \Omega_{Y_{1}}\right)$, no pattern with 101 exists.

Lemma 2. The number of the pattern 11 in $f_{1} \in \Omega_{Y_{1}}$ is equal to that of the pattern 111 in $I_{1} f_{1}$, and it does not depend on the specific state $f_{1}$. If we denote it by $\mathcal{N}_{2}$, it is calculated as

$$
\mathcal{N}_{2}=\sum_{i=3}^{P_{1}} k_{i}(i-2)
$$

Then we have the following theorem.

\section{Theorem 1.}

$$
C_{Y}(1,2, \ldots, r+1)=\left(\frac{L_{1}}{L} \prod_{i=1}^{r} \frac{\left(L_{1}-i\right)}{\left(L_{1}+k_{1}-i\right)}\right) C_{Y_{1}}(1,2, \ldots, r) \quad(r=1,2, \ldots) .
$$


In particular,

$$
\begin{aligned}
& C_{Y}(1,2)=\frac{\left(L_{1}-1\right)}{L\left(L_{1}+k_{1}-1\right)} \sum_{i=3}^{P_{1}} k_{i}(i-2), \\
& C_{Y}(1,2,3)=\frac{L_{1}\left(L_{1}-1\right)\left(L_{1}-2\right)}{L\left(L_{1}+k_{1}-1\right)\left(L_{1}+k_{1}-2\right)} C_{Y_{1}}(1,2) .
\end{aligned}
$$

Proof. We call a 'block' for a repeated 10 pattern of the form $\underline{10} \underline{10} \cdots \underline{10}$. We also call a 111 pattern for the pattern of the form 111.

First let us consider the case $r=1$. From Lemma 2, the number of 111 patterns in $I_{1} f_{1}$ is equal to $\sum_{i=3}^{P_{1}} k_{i}(i-2)$. Let $\mathcal{B}_{1}$ be a set $\left\{B_{1} \in\left(\mathbb{Z}_{\geq 0}\right)^{L_{1}} \mid \sum_{i=1}^{L_{1}} b_{i}=k_{1}, B_{1}=\left(b_{1}, b_{2}, \ldots, b_{L_{1}}\right)\right\}$. In the process $I_{1} f_{1} \mapsto I\left(B_{1}\right) f_{1}$, if $\underline{10}$ blocks is inserted between the left 1 and the middle 1 of a 111 pattern, then the 111 pattern in $I\left(B_{1}\right) f_{1}$ disappears. Let $\Psi_{1}: \mathbb{Z}_{\geq 0} \times\left(\mathbb{F}_{2} \times \mathbb{F}_{2}\right) \rightarrow\{0,1\}$ be the function:

$$
\Psi_{1}\left(b ; u_{1}, u_{2}\right):= \begin{cases}1 & \left(b=0,\left(u_{1}, u_{2}\right)=(1,1)\right), \\ 0 & \text { otherwise }\end{cases}
$$

and $\psi_{1}\left(f_{1}\right):=\sharp\left\{i \in\left[L_{1}\right] \mid\left(f_{1}(i), f_{1}(i+1)\right)=(1,1)\right\}$. Since a state of $\Omega_{Y}$ is obtained by 10 -insertion to a state in $\Omega_{Y_{1}}$, we have

$$
\begin{aligned}
C_{Y}(1,2) & =\frac{1}{L\left|\mathcal{B}_{1}\right|\left|\Omega_{Y_{1}}\right|} \sum_{B_{1} \in \mathcal{B}_{1}} \sum_{f_{1} \in \Omega_{Y_{1}}} \sum_{i=1}^{L}\left(I\left(B_{1}\right) f_{1}\right)(i)\left(I\left(B_{1}\right) f_{1}\right)(i+1)\left(I\left(B_{1}\right) f_{1}\right)(i+2) \\
& =\frac{1}{L\left|\mathcal{B}_{1}\right|\left|\Omega_{Y_{1}}\right|} \sum_{B_{1} \in \mathcal{B}_{1}} \sum_{f_{1} \in \Omega_{Y_{1}}} \sum_{i=1}^{L_{1}} \Psi_{1}\left(b_{i} ; f_{1}(i), f_{1}(i+1)\right) \\
& =\frac{1}{L\left|\mathcal{B}_{1}\right|\left|\Omega_{Y_{1}}\right|} \sum_{B_{1} \in \mathcal{B}_{1}} \sum_{f_{1} \in \Omega_{Y_{1}}} \sum_{i=1}^{L_{1}} \Psi_{1}\left(b_{1} ; f_{1}(i), f_{1}(i+1)\right) \\
& =\frac{1}{L\left|\mathcal{B}_{1}\right|\left|\Omega_{Y_{1}}\right|} \sum_{B_{1} \in \mathcal{B}_{1}} \sum_{f_{1} \in \Omega_{Y_{1}}} \psi_{1}\left(f_{1}\right) \Psi_{1}\left(b_{1} ; 1,1\right) \\
& =\frac{1}{L\left|\mathcal{B}_{1}\right|\left|\Omega_{Y_{1}}\right|} \sum_{f_{1} \in \Omega_{Y_{1}}} \psi_{1}\left(f_{1}\right) \sum_{B_{1} \in \mathcal{B}_{1}} \Psi_{1}\left(b_{1} ; 1,1\right) \\
& =\frac{\left(\begin{array}{c}
L_{1}+k_{1}-2 \\
k_{1}
\end{array}\right)}{L\left|\mathcal{B}_{1}\right|}\left(\frac{1}{\left|\Omega_{Y_{1}}\right|} \sum_{f_{1} \in \Omega_{Y_{1}}} \psi_{1}\left(f_{1}\right)\right)=\frac{\left(L_{1}-1\right)}{L\left(L_{1}+k_{1}-1\right)} \sum_{i=3}^{P_{1}} k_{i}(i-2) .
\end{aligned}
$$

From

$$
\sum_{i=3}^{P_{1}} k_{i}(i-2)=\sum_{i=2}^{P_{1}} k_{i}(i-1)-\hat{k}_{2}=L_{1} C_{Y_{1}}(1),
$$

we find

$$
C_{Y}(1,2)=\frac{L_{1}}{L} \frac{\left(L_{1}-1\right)}{\left(L_{1}+k_{1}-1\right)} C_{Y_{1}}(1) .
$$

For general $r$, if we define

$$
\Psi_{r}\left(b_{1}, b_{2}, \ldots, b_{r} ; u_{1}, u_{2}, \ldots, u_{r+1}\right)
$$




$$
:= \begin{cases}1 & \left(\left(b_{1}, b_{2}, \ldots, b_{r}\right)=(0,0, \ldots, 0),\left(u_{1}, u_{2}, \ldots, u_{r+1}\right)=(1,1, \ldots, 1)\right), \\ 0 & \text { otherwise }\end{cases}
$$

and $\psi_{r}\left(f_{1}\right):=\sharp\left\{i \in\left[L_{1}\right] \mid\left(f_{1}(i), f_{1}(i+1), \ldots, f_{1}(i+r)\right)=(1,1, \ldots, 1)\right\}$, we can proceed in a similar manner to the case $r=1$ as

$$
\begin{aligned}
C_{Y}(1,2, \ldots, r+1) & =\frac{1}{L\left|\mathcal{B}_{1}\right|\left|\Omega_{Y_{1}}\right|} \sum_{B_{r} \in \mathcal{B}_{1}} \sum_{f_{1} \in \Omega_{Y_{1}}} \sum_{i=1}^{L}\left(I\left(B_{1}\right) f_{1}\right)(i) \ldots\left(I\left(B_{1}\right) f_{1}\right)(i+r+1) \\
& =\frac{1}{L\left|\mathcal{B}_{1}\right|\left|\Omega_{Y_{1}}\right|} \sum_{B_{r} \in \mathcal{B}_{1}} \sum_{f_{1} \in \Omega_{Y_{1}}} \sum_{i=1}^{L_{1}} \Psi_{r}\left(b_{i}, \ldots, b_{i+r} ; f_{1}(i), \ldots, f_{1}(i+r+1)\right) \\
& =\frac{1}{L\left|\mathcal{B}_{1}\right|\left|\Omega_{Y_{1}}\right|} \sum_{B_{r} \in \mathcal{B}_{1}} \sum_{f_{1} \in \Omega_{Y_{1}}} \sum_{i=1}^{L_{1}} \Psi_{r}\left(b_{1}, \ldots, b_{r+1} ; f_{1}(i), \ldots, f_{1}(i+r+1)\right) \\
& =\frac{1}{L\left|\mathcal{B}_{1}\right|\left|\Omega_{Y_{1}}\right|} \sum_{B_{1} \in \mathcal{B}_{1}} \sum_{f_{1} \in \Omega_{Y_{1}}} \psi_{r}\left(f_{1}\right) \Psi_{r}\left(b_{1}, \cdots, b_{r+1} ;(1,1, \ldots, 1)\right) \\
& =\frac{\left(\begin{array}{c}
L_{1}+k_{1}-r-1 \\
k_{1}
\end{array}\right)}{L\left|\mathcal{B}_{1}\right|} \times\left(\frac{1}{\left|\Omega_{Y_{1}}\right|} \sum_{f_{1} \in \Omega_{Y_{1}}} \psi_{r}\left(f_{1}\right)\right) \\
& =\frac{\left(\begin{array}{c}
L_{1}+k_{1}-r-1 \\
k_{1}
\end{array}\right)}{L\left(\begin{array}{c}
L_{1}+k_{1}-1 \\
k_{1}
\end{array}\right)} \times L_{1} C_{Y_{1}}(1,2, \ldots, r) \\
& =\frac{L_{1} C_{Y_{1}}(1,2, \ldots, r)}{L} \prod_{i=1}^{r} \frac{\left(L_{1}-i\right)}{\left(L_{1}+k_{1}-i\right)}
\end{aligned}
$$

which completes the proof.

\section{The second and the third nearest neighbor correlation functions}

\subsection{Correlation function $C_{Y}(2)$}

Using 10-insertion and the Young diagram $Y_{1}$ defined in the previous section, we can rewrite $C_{Y}(2)$ as

$$
C_{Y}(2)=\frac{1}{L\left|\mathcal{B}_{1}\right|\left|\Omega_{Y_{1}}\right|} \sum_{B_{1} \in \mathcal{B}_{1}} \sum_{f_{1} \in \Omega_{Y_{1}}} \sum_{n=1}^{L}\left(I\left(B_{1}\right) f_{1}\right)(n)\left(I\left(B_{1}\right) f_{1}\right)(n+2) .
$$

From this expression we can prove the following proposition which gives a key formula to calculate $C_{Y}(2)$.

Proposition 1. Let $\Phi_{0}: \mathbb{Z}_{\geq 0} \times\left(\mathbb{F}_{2} \times \mathbb{F}_{2}\right) \rightarrow \mathbb{Z}_{\geq 0}$ be the function defined by

$$
\Phi_{0}\left(b ; u_{1}, u_{2}\right):= \begin{cases}0 & (b=0), \\ b & \left(b \neq 0,\left(u_{1}, u_{2}\right)=(0,1),(1,0)\right), \\ b-1 & \left(b \neq 0,\left(u_{1}, u_{2}\right)=(0,0),(1,1)\right) .\end{cases}
$$

Then we have

$$
C_{Y}(2)=\frac{1}{L}\left(\mathcal{N}_{2}+\mathcal{R}_{2}\right),
$$


where $\mathcal{N}_{2}$ is given in (1),

$$
\mathcal{R}_{2}=\frac{1}{\left|\mathcal{B}_{1}\right|} \sum_{B_{1} \in \mathcal{B}_{1}} \sum_{\boldsymbol{b} \in\left(\mathbb{F}_{2}\right)^{2}} \phi_{0}\left(f_{1} ; \boldsymbol{b}\right) \Phi_{0}\left(b_{1} ; \boldsymbol{b}\right) \quad\left(f_{1} \in \Omega_{Y_{1}}\right),
$$

and $\phi_{0}\left(f_{1} ; \boldsymbol{b}\right):=\sharp\left\{i \in\left[L_{1}\right] \mid\left(f_{1}(i), f_{1}(i+1)\right)=\boldsymbol{b}\right\}$.

Proof. We call a $1 * 1$ pattern for the pattern of the form $1 * 1$ where $*$ is either 1 or 0 . From Lemmas 1 and 2, we already know the number of $1 * 1$ patterns in $I_{1} f_{1}$. Hence we estimate the number of the $1 * 1$ patterns generated and eliminated by inserting blocks $\underline{10} \underline{10} \cdots \underline{10}$ in the process $I_{1} f_{1} \mapsto I\left(B_{1}\right) f_{1}$. Since there appears no $1 * 1$ pattern between blocks, we have only to count the number of the patterns 101 appeared in a block and a boundary and that of the patterns 111 eliminated by 10 -insertion; then, for $b_{n} \geq 1$, we observe the number of $1 * 1$ patterns appeared by the operation of $I_{2}\left(B_{1}\right)$ as

(1) $f_{1}(n) f_{1}(n+1)=\mathbf{0 0} \longrightarrow \mathbf{0 1 0} \underline{10} \cdots \underline{100} \Rightarrow+\left(b_{n}-1\right)$,

(2) $f_{1}(n) f_{1}(n+1)=\mathbf{1 0} \longrightarrow \mathbf{1} 10 \underline{10} \underline{10} \cdots \underline{100} \Rightarrow+b_{n}$,

(3) $f_{1}(n) f_{1}(n+1)=\mathbf{0 1} \longrightarrow \mathbf{0 1 0} \underline{10} \cdots \underline{101} \Rightarrow+b_{n}$,

(4) $f_{1}(n) f_{1}(n+1)=\mathbf{1 1} \longrightarrow \mathbf{1} \underline{10} \underline{10} \cdots \underline{101} \Rightarrow+\left(b_{n}-1\right)$.

Here we write the referred 1,0 s in $I_{1} f_{1}$ in bold scripts $(\mathbf{1}, \mathbf{0})$ for clarifying the results. Note that if $f_{1}(n) f_{1}(n+1)=\mathbf{1 1}$ and $b_{n}=0$, then we have the patterns $\mathbf{1 1 1}$ or $\mathbf{1 1} \underline{10}$ or $\mathbf{1 1} \underline{10}$ after the operation of $I_{2}\left(B_{1}\right)$. Hence, we have

$$
\begin{aligned}
\mathcal{R}_{2} & =\frac{1}{\left|\mathcal{B}_{1}\right|\left|\Omega_{Y_{1}}\right|} \sum_{B_{1} \in \mathcal{B}_{1}} \sum_{f_{1} \in \Omega_{Y_{1}}}\left(\sharp\left\{{ }^{\prime} 1011^{\prime} \in I\left(B_{1}\right) f_{1}\right\}-\sharp\left\{{ }^{\prime} 111^{\prime} \in I_{1} f_{1} \text { disappears in } I\left(B_{1}\right) f_{1}\right\}\right) \\
& =\frac{1}{\left|\mathcal{B}_{1}\right|\left|\Omega_{Y_{1}}\right|} \sum_{B_{1} \in \mathcal{B}_{1}} \sum_{f_{1} \in \Omega_{Y_{1}}} \sum_{i=1}^{L_{1}} \Phi_{0}\left(b_{i} ; f_{1}(i), f_{1}(i+1)\right) .
\end{aligned}
$$

Here $\sharp\left\{\right.$ ' 101 ' $\left.\in I\left(B_{1}\right) f_{1}\right\}$ denotes the number of ' 101 's in $I\left(B_{1}\right) f_{1}$ and $\sharp\left\{\right.$ ' 111 ' $\in I_{1} f_{1}$ disappears in $\left.I\left(B_{1}\right) f_{1}\right\}$ denotes the number of ' 101 's which disappear by the operation $I_{2}\left(B_{1}\right)$. Since

$$
\left(b_{1}, b_{2}, \ldots, b_{L_{1}}\right) \in \mathcal{B}_{1} \longrightarrow\left(b_{i}, b_{i+1}, \ldots, b_{L_{1}}, b_{1}, \ldots, b_{i-1}\right) \in \mathcal{B}_{1},
$$

we have

$$
\sum_{B_{1} \in \mathcal{B}_{1}} \Phi_{0}\left(b_{i} ; f_{1}(i), f_{1}(i+1)\right)=\sum_{B_{1} \in \mathcal{B}_{1}} \Phi_{0}\left(b_{1} ; f_{1}(i), f_{1}(i+1)\right) .
$$

Thus we have

$$
\begin{aligned}
\sum_{B_{1} \in \mathcal{B}_{1}} \sum_{i=1}^{L_{1}} \Phi_{0}\left(b_{i} ; f_{1}(i), f_{1}(i+1)\right) & =\sum_{B_{1} \in \mathcal{B}_{1}} \sum_{i=1}^{L_{1}} \Phi_{0}\left(b_{1} ; f_{1}(i), f_{1}(i+1)\right) \\
& =\sum_{B_{1} \in \mathcal{B}_{1}} \sum_{\boldsymbol{b} \in\left(\mathbb{F}_{2}\right)^{2}} \phi_{0}\left(f_{1} ; \boldsymbol{b}\right) \Phi_{0}\left(b_{1}, \boldsymbol{b}\right) .
\end{aligned}
$$

Furthermore, if $f$ and $f^{\prime}$ are the states of PBBS with the same number of boxes, balls and solitons, then $\phi_{0}(f ; \boldsymbol{b})=\phi_{0}\left(f^{\prime} ; \boldsymbol{b}\right)$ for any $\boldsymbol{b} \in\left(\mathbb{F}_{2}\right)^{2}$, in particular, $\phi_{0}\left(f_{1} ; \boldsymbol{b}\right)=\phi_{0}\left(f_{1}^{\prime} ; \boldsymbol{b}\right)$ for $f_{1}, f_{1}^{\prime} \in \Omega_{Y_{1}}$. Therefore we obtain (3). 
For the evaluation of $C_{Y}(2)$, we use the identity for binomial coefficients given in the following lemma.

Lemma 3. For $k \leq l \leq n$, it holds that

$$
\left(\begin{array}{l}
n \\
l
\end{array}\right)=\sum_{m=0}^{n-l}\left\{\left(\begin{array}{c}
k+m \\
k
\end{array}\right)-\left(\begin{array}{c}
k+m-1 \\
k
\end{array}\right)\right\}\left(\begin{array}{c}
n-k-m \\
l-k
\end{array}\right)
$$

\section{Theorem 2.}

$$
\begin{aligned}
C_{Y}(2) & =\frac{1}{L}\left(\sum_{i=3}^{P_{1}} k_{i}(i-2)+\frac{k_{1}\left(k_{1}-1\right)}{L_{1}+k_{1}-2}+\frac{2 \hat{k}_{2} k_{1}}{L_{1}+k_{1}-1}\right) \\
& =\frac{1}{L}\left(L_{1} C_{Y_{1}}(1)+\frac{k_{1}\left(k_{1}-1\right)}{L_{1}+k_{1}-2}+\frac{2 \hat{k}_{2} k_{1}}{L_{1}+k_{1}-1}\right) .
\end{aligned}
$$

Proof. There are $\left(\begin{array}{c}L_{1}+k_{1}-m-2 \\ k_{1}-m\end{array}\right)$ elements with $b_{1}=m(m \geq 1)$ in the set $\mathcal{B}_{1}$. Since $\phi_{0}\left(f_{1} ;(0,1)\right)$ $=\phi_{0}\left(f_{1} ;(1,0)\right)=\hat{k}_{2}$ and $\phi_{0}\left(f_{1} ;(0,0)\right)+\phi_{0}\left(f_{1} ;(1,1)\right)=L_{1}-2 \hat{k}_{2}$, we have

$$
\begin{aligned}
& \sum_{B_{1} \in \mathcal{B}_{1}} \sum_{\boldsymbol{b} \in\left(\mathbb{F}_{2}\right)^{2}} \phi_{0}\left(f_{1} ; \boldsymbol{b}\right) \Phi_{0}\left(b_{1} ; \boldsymbol{b}\right)=\sum_{m=1}^{k_{1}}\left(\begin{array}{c}
L_{1}+k_{1}-m-2 \\
k_{1}-m
\end{array}\right) \sum_{\boldsymbol{b} \in\left(\mathbb{F}_{2}\right)^{2}} \phi_{0}\left(f_{1} ; \boldsymbol{b}\right) \Phi_{0}(m ; \boldsymbol{b}) \\
& =\sum_{m=1}^{k_{1}}\left(\begin{array}{c}
L_{1}+k_{1}-m-2 \\
k_{1}-m
\end{array}\right)\left(\sum_{\boldsymbol{b}=(0,0),(1,1)}(m-1) \phi_{0}\left(f_{1} ; \boldsymbol{b}\right)+\sum_{\boldsymbol{b}=(0,1),(1,0)} m \phi_{0}\left(f_{1} ; \boldsymbol{b}\right)\right) \\
& =L_{1} \sum_{m=1}^{k_{1}}(m-1)\left(\begin{array}{c}
L_{1}+k_{1}-m-2 \\
k_{1}-m
\end{array}\right)+2 \hat{k}_{2}\left(\begin{array}{c}
L_{1}+k_{1}-2 \\
k_{1}-1
\end{array}\right),
\end{aligned}
$$

that is,

$$
C_{Y}(2)=\frac{1}{L}\left(\sum_{i=3}^{P_{1}} k_{i}(i-2)+\frac{L_{1} \sum_{m=1}^{k_{1}}(m-1)\left(\begin{array}{c}
L_{1}+k_{1}-m-2 \\
k_{1}-m
\end{array}\right)}{\left|\mathcal{B}_{1}\right|}+\frac{2 \hat{k}_{2}\left(\begin{array}{c}
L_{1}+k_{1}-2 \\
k_{1}-1
\end{array}\right)}{\left|\mathcal{B}_{1}\right|}\right) .
$$

When we put $n=L_{1}+k_{1}-2$ and $l=L_{1}$, (4) turns into

$$
\left(\begin{array}{c}
L_{1}+k_{1}-2 \\
L_{1}
\end{array}\right)=\sum_{m=0}^{k_{1}-2}(m+1)\left(\begin{array}{c}
L_{1}+k_{1}-2-2-m \\
k_{1}-2-m
\end{array}\right)=\sum_{m=1}^{k_{1}}(m-1)\left(\begin{array}{c}
L_{1}+k_{1}-2-m \\
k_{1}-m
\end{array}\right) .
$$

Since $\left|\mathcal{B}_{1}\right|=\left(\begin{array}{c}L_{1}+k_{1}-1 \\ k_{1}\end{array}\right)$, we obtain (5). Equation (6) follows from (2).

\subsection{Correlation function $C_{Y}(3)$}

The evaluation of $C_{Y}(3)$ can be done in analogous way to that of $C_{Y}(2)$, although we have to consider various patterns so that the expression may become fairly complicated.

Using 10-insertion and the Young diagram $Y_{2}$ defined in the previous section, we have

$$
C_{Y}(3)=\frac{1}{L\left|\mathcal{B}_{1}\right|\left|\mathcal{B}_{2}\right|\left|\Omega_{Y_{2} \mid}\right|} \sum_{B_{1} \in \mathcal{B}_{1}} \sum_{B_{2} \in \mathcal{B}_{2}} \sum_{f_{2} \in \Omega_{Y_{2}}}
$$




$$
\sum_{n=1}^{L}\left(I\left(B_{1}\right) I\left(B_{2}\right) f_{2}\right)(n)\left(I\left(B_{1}\right) I\left(B_{2}\right) f_{2}\right)(n+3)
$$

where $\mathcal{B}_{2}=\left\{B_{2} \in\left(\mathbb{Z}_{\geq 0}\right)^{L_{2}} \mid \sum_{i=1}^{L_{2}} b_{i}=k_{2}, B_{2}=\left(b_{1}, b_{2}, \ldots, b_{L_{2}}\right)\right\}$. We call a $1 * * 1$ pattern for an array of 4 elements of the form $1 * * 1$ where $* *$ is 00 or 01 or 10 or 11 . The following lemmas are easily understood by the definition of 10-insertion and Proposition 1.

Lemma 4. In a state $f=I_{1} f_{1}\left(f_{1} \in \Omega_{Y_{1}}\right)$, no pattern with 1101 and 1011 exists.

Lemma 5. For $B_{2} \in \mathcal{B}_{2}$ and $f_{2} \in \Omega_{Y_{2}}$, the number of the $1 * 1$ patterns in $f_{1}:=I\left(B_{2}\right) f_{2}$ is equal to that of the patterns of the form 1001 and 1111 in $I_{1} f_{1}$, and is given by

$$
\sum_{i=4}^{P_{1}} k_{i}(i-3)+j\left(B_{2}\right)
$$

where

$$
j\left(B_{2}\right):=\sum_{i=1}^{L_{2}} \Phi_{0}\left(\tilde{b}_{i} ; f_{2}(i), f_{2}(i+1)\right) \quad\left(B_{2}=\left(\tilde{b}_{1}, \tilde{b}_{2}, \ldots, \tilde{b}_{L_{2}}\right) \in \mathcal{B}_{2}\right) .
$$

Lemma 6. Let $\widetilde{\mathcal{N}}_{2}:=\sum_{i=4}^{P_{1}} k_{i}(i-3)$ and $\widetilde{\mathcal{R}}_{2}:=\left.\mathcal{R}_{2}\right|_{\left(f_{1}, \mathcal{B}_{1}\right) \rightarrow\left(f_{2}, \mathcal{B}_{2}\right)}=\frac{1}{\left|\mathcal{B}_{2}\right|} \sum_{B_{2} \in \mathcal{B}_{2}} j\left(B_{2}\right)$. Then we have

$$
C_{Y_{1}}(2)=\frac{1}{L_{1}}\left(\widetilde{\mathcal{N}}_{2}+\widetilde{\mathcal{R}}_{2}\right)
$$

Let us define

$$
\mathcal{N}_{3}:=\frac{1}{\left|\mathcal{B}_{1}\right|\left|\mathcal{B}_{2}\right|\left|\Omega_{Y_{2}}\right|} \sum_{B_{1} \in \mathcal{B}_{1}} \sum_{B_{2} \in \mathcal{B}_{2}} \sum_{f_{2} \in \Omega_{Y_{2}}} \sharp\left\{' 1111 \text { ', '1001' } \in I_{1} I\left(B_{2}\right) f_{2}\right\} .
$$

From Lemmas $4-6$, we find $\mathcal{N}_{3}=L_{1} C_{Y_{1}}(2)$. If we define $\mathcal{R}_{3}$ by

$$
C_{Y}(3)=\frac{1}{L}\left(\mathcal{N}_{3}+\mathcal{R}_{3}\right)
$$

it denotes the term which arises from 10-insertion $I\left(B_{1}\right)$, that is,

$$
\begin{aligned}
& \mathcal{R}_{3}=\frac{1}{\left|\mathcal{B}_{1}\right|\left|\mathcal{B}_{2}\right|\left|\Omega_{Y_{2}}\right|} \sum_{B_{1} \in \mathcal{B}_{1}} \sum_{B_{2} \in \mathcal{B}_{2}} \sum_{f_{2} \in \Omega_{Y_{2}}}\left(\sharp\left\{{ }^{\prime} 1101 ', ' 1011 \text { ' } \in I\left(B_{1}\right) I\left(B_{2}\right) f_{2}\right\}\right. \\
& \left.-\sharp\left\{\text { ' } 1001 \text { ', ' } 1111 \text { ' } \in I_{1} I\left(B_{2}\right) f_{2} \text { disappear in } I\left(B_{1}\right) I\left(B_{2}\right) f_{2}\right\}\right) \text {. }
\end{aligned}
$$

Then we obtain the following proposition which gives a key formula to calculate $C_{Y}(3)$.

Proposition 2. Let us define the functions; $\Phi_{1}: \mathbb{Z}_{\geq 0} \times\left(\mathbb{F}_{2}\right)^{4} \rightarrow\{0,1,2\}$ :

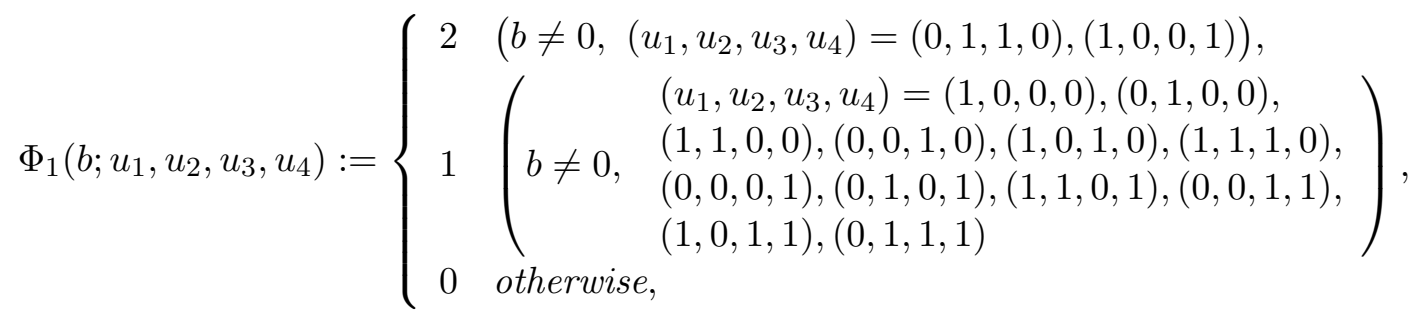


$\Phi_{2}:\left(\mathbb{Z}_{\geq 0}\right)^{2} \times\left(\mathbb{F}_{2}\right)^{4} \rightarrow\{-1,0,1\}:$

$$
\Phi_{2}\left(b, b^{\prime} ; u_{1}, u_{2}, u_{3}, u_{4}\right):=\left\{\begin{array}{rll}
1 & \left(b b^{\prime} \neq 0,\right. & \left.\left(u_{1}, u_{2}, u_{3}, u_{4}\right)=(1,0,0,0),(0,0,0,0)\right), \\
-1 & \left(b b^{\prime} \neq 0,\right. & \left(u_{1}, u_{2}, u_{3}, u_{4}\right)=(1,1,0,1),(0,1,0,1), \\
0 & \text { otherwise, }
\end{array}\right.
$$

and $\Phi_{3}:\left(\mathbb{Z}_{\geq 0}\right)^{2} \times\left(\mathbb{F}_{2}\right)^{4} \rightarrow\{-1,0\}:$

$$
\Phi_{3}\left(b, b^{\prime} ; u_{1}, u_{2}, u_{3}, u_{4}\right):=\left\{\begin{aligned}
-1 & \left(b b^{\prime} \neq 0,\left(u_{1}, u_{2}, u_{3}, u_{4}\right)=(1,1,1,1)\right) \\
0 & \text { otherwise. }
\end{aligned}\right.
$$

Then we have

$$
\begin{aligned}
& C_{Y}(3)=\frac{1}{L}\left(\mathcal{N}_{3}+\mathcal{R}_{3}\right), \\
& \mathcal{N}_{3}=L_{1} C_{Y_{1}}(2), \\
& \mathcal{R}_{3}=\frac{1}{\left|\mathcal{B}_{2}\right|\left|\mathcal{B}_{1}\right|\left|\Omega_{Y_{2}}\right|} \sum_{B_{2} \in \mathcal{B}_{2}} \sum_{B_{1} \in \mathcal{B}_{1}} \sum_{f_{2} \in \Omega_{Y_{2}}} \sum_{\boldsymbol{b} \in\left(\mathbb{F}_{2}\right)^{4}} \\
& \quad \phi_{1}\left(I\left(B_{2}\right) f_{2} ; \boldsymbol{b}\right)\left(\Phi_{1}\left(b_{1} ; \boldsymbol{b}\right)+\Phi_{2}\left(b_{1}, b_{2} ; \boldsymbol{b}\right)+\Phi_{3}\left(b_{1}, b_{3} ; \boldsymbol{b}\right)\right),
\end{aligned}
$$

where

$$
\phi_{1}\left(f_{1} ; \boldsymbol{b}\right):=\sharp\left\{i \in\left[L_{1}\right] \mid\left(f_{1}(i-1), f_{1}(i), f_{1}(i+1), f_{1}(i+2)\right)=\boldsymbol{b}\right\} .
$$

Proof. Equations (10) and (11) have already been shown. From (17) in Appendix A, we find that

$$
\begin{aligned}
\mathcal{R}_{3}= & \frac{1}{\left|\mathcal{B}_{1}\right|\left|\mathcal{B}_{2}\right|\left|\Omega_{Y_{2}}\right|} \sum_{B_{2} \in \mathcal{B}_{2}} \sum_{B_{1} \in \mathcal{B}_{1}} \sum_{f_{2} \in \Omega_{Y_{2}}} \sum_{i=1}^{L_{1}} \\
& \left(\Phi_{1}\left(b_{i} ; I\left(B_{2}\right) f_{2}(i-1), I\left(B_{2}\right) f_{2}(i), I\left(B_{2}\right) f_{2}(i+1), I\left(B_{2}\right) f_{2}(i+2)\right)\right. \\
& +\Phi_{2}\left(b_{i}, b_{i+1} ; I\left(B_{2}\right) f_{2}(i-1), I\left(B_{2}\right) f_{2}(i), I\left(B_{2}\right) f_{2}(i+1), I\left(B_{2}\right) f_{2}(i+2)\right) \\
& \left.+\Phi_{3}\left(b_{i-1}, b_{i+1} ; I\left(B_{2}\right) f_{2}(i-1), I\left(B_{2}\right) f_{2}(i), I\left(B_{2}\right) f_{2}(i+1), I\left(B_{2}\right) f_{2}(i+2)\right)\right) .
\end{aligned}
$$

Since

$$
\left(b_{1}, b_{2}, \ldots, b_{L_{1}}\right) \in \mathcal{B}_{1} \longrightarrow\left(b_{i}, b_{i}+1, \ldots, b_{L_{1}}, b_{1}, \ldots, b_{i-1}\right) \in \mathcal{B}_{1}
$$

we can rewrite

$$
\begin{aligned}
\sum_{B_{1} \in \mathcal{B}_{1}} & \Phi_{1}\left(b_{i} ; I\left(B_{2}\right) f_{2}(i-1), I\left(B_{2}\right) f_{2}(i), I\left(B_{2}\right) f_{2}(i+1), I\left(B_{2}\right) f_{2}(i+2)\right) \\
& =\sum_{B_{1} \in \mathcal{B}_{1}} \Phi_{1}\left(b_{1} ; I\left(B_{2}\right) f_{2}(i-1), I\left(B_{2}\right) f_{2}(i), I\left(B_{2}\right) f_{2}(i+1), I\left(B_{2}\right) f_{2}(i+2)\right) .
\end{aligned}
$$

Furthermore

$$
\sum_{i=1}^{L_{1}} \Phi_{1}\left(b_{1} ; I\left(B_{2}\right) f_{2}(i-1), I\left(B_{2}\right) f_{2}(i), I\left(B_{2}\right) f_{2}(i+1), I\left(B_{2}\right) f_{2}(i+2)\right)
$$




$$
=\sum_{\boldsymbol{b} \in\left(\mathbb{F}_{2}\right)^{4}} \phi_{1}\left(I\left(B_{2}\right) f_{2} ; \boldsymbol{b}\right) \Phi_{1}\left(b_{1} ; \boldsymbol{b}\right) .
$$

Similar relations hold for $\Phi_{2}$ and $\Phi_{3}$, and we obtain

$$
\begin{aligned}
\mathcal{R}_{3}= & \frac{1}{\left|\mathcal{B}_{1}\right|\left|\mathcal{B}_{2}\right|\left|\Omega_{Y_{2}}\right|} \sum_{B_{2} \in \mathcal{B}_{2}} \sum_{B_{1} \in \mathcal{B}_{1}} \sum_{f_{2} \in \Omega_{Y_{2}}} \sum_{\boldsymbol{b} \in\left(\mathbb{F}_{2}\right)^{4}} \\
& \phi_{1}\left(I\left(B_{2}\right) f_{2} ; \boldsymbol{b}\right)\left(\Phi_{1}\left(b_{1} ; \boldsymbol{b}\right)+\Phi_{2}\left(b_{1}, b_{2} ; \boldsymbol{b}\right)+\Phi_{3}\left(b_{1}, b_{3} ; \boldsymbol{b}\right)\right) .
\end{aligned}
$$

\section{Theorem 3.}

$$
\begin{aligned}
C_{Y}(3)= & \frac{1}{L}\left(L_{1} C_{Y_{1}}(2)+2 K_{1}^{(1)}\left(3 \hat{k}_{3}+2 k_{2}-\widetilde{\mathcal{R}}_{2}\right)\right. \\
& +K_{1}^{(1)} K_{2}^{(1)}\left\{L_{1}-4 \hat{k}_{3}-3 k_{2}-\widetilde{\mathcal{R}}_{2}-\left(K_{1}^{(2)}+1\right) L_{2} C_{Y_{2}}(1)-L_{1} C_{Y_{1}}(1,2,3)\right\} \\
& \left.-K_{1}^{(1)} K_{2}^{(1)} K_{3}^{(1)} \frac{\left(L_{1}-1\right) L_{1} C_{Y_{1}}(1,2,3)}{k_{1}-2}\right),
\end{aligned}
$$

where

$$
K_{i}^{(j)}:=\frac{k_{j}-i+1}{L_{j}+k_{j}-i},
$$

and

$$
\widetilde{\mathcal{R}}_{2}=\frac{2 \hat{k}_{3} k_{2}}{L_{2}+k_{2}-1}+\frac{k_{2}\left(k_{2}-1\right)}{L_{2}+k_{2}-2} .
$$

Proof. From (18) and (19) in Appendix B, we obtain the following equations:

$$
\begin{aligned}
& \sum_{B_{1} \in \mathcal{B}_{1}} \sum_{\boldsymbol{b} \in\left(\mathbb{F}_{2}\right)^{4}} \phi_{1}\left(I\left(B_{2}\right) f_{2} ; \boldsymbol{b}\right) \Phi_{1}\left(b_{1} ; \boldsymbol{b}\right)=\left(\begin{array}{c}
L_{1}+k_{1}-2 \\
k_{1}-1
\end{array}\right)\left\{2\left(3 \hat{k}_{3}+2 k_{2}-j\left(B_{2}\right)\right)\right\}, \\
& \sum_{B_{1} \in \mathcal{B}_{1}}\left(\phi_{1}\left(I\left(B_{2}\right) f_{2} ; 1,0,0,0\right) \Phi_{2}\left(b_{1}, b_{2} ; 1,0,0,0\right)+\phi_{1}\left(I\left(B_{2}\right) f_{2} ; 0,0,0,0\right) \Phi_{2}\left(b_{1}, b_{2} ; 0,0,0,0\right)\right. \\
& \left.+\phi_{1}\left(I\left(B_{2}\right) f_{2} ; 1,1,0,1\right) \Phi_{2}\left(b_{1}, b_{2} ; 1,1,0,1\right)+\phi_{1}\left(I\left(B_{2}\right) f_{2} ; 0,1,0,1\right) \Phi_{2}\left(b_{1}, b_{2} ; 0,1,0,1\right)\right) \\
& =\left(\begin{array}{c}
L_{1}+k_{1}-3 \\
k_{1}-2
\end{array}\right)\left(\phi_{1}\left(I\left(B_{2}\right) f_{2} ; 1,0,0,0\right)+\phi_{1}\left(I\left(B_{2}\right) f_{2} ; 0,0,0,0\right)\right. \\
& \left.-\phi_{1}\left(I\left(B_{2}\right) f_{2} ; 1,1,0,1\right)-\phi_{1}\left(I\left(B_{2}\right) f_{2} ; 0,1,0,1\right)\right) \\
& =\left(\begin{array}{c}
L_{1}+k_{1}-3 \\
k_{1}-2
\end{array}\right)\left(L_{1}-4 \hat{k}_{3}-3 k_{2}-j\left(B_{2}\right)-\sum_{i=1}^{L_{2}} \zeta\left(\tilde{b}_{i} ; f_{2}(i), f_{2}(i+1)\right)-\sum_{i=4}^{P_{1}} k_{i}(i-3)\right), \\
& \sum_{B_{1} \in \mathcal{B}_{1}} \phi_{1}\left(I\left(B_{2}\right) f_{2} ; 1,1,1,1\right)\left(\Phi_{2}\left(b_{1}, b_{2} ; 1,1,1,1\right)+\Phi_{3}\left(b_{1}, b_{3} ; 1,1,1,1\right)\right) \\
& =-\left\{\left(\begin{array}{c}
L_{1}+k_{1}-3 \\
k_{1}-2
\end{array}\right)+\left(\begin{array}{c}
L_{1}+k_{1}-4 \\
k_{1}-2
\end{array}\right)\right\} \phi_{1}\left(I\left(B_{2}\right) f_{2} ; 1,1,1,1\right),
\end{aligned}
$$

where $\left(\tilde{b}_{1}, \tilde{b}_{2}, \ldots, \tilde{b}_{L_{2}}\right)=B_{2} \in \mathcal{B}_{2}$ and

$$
\zeta\left(b ; u_{1}, u_{2}\right):= \begin{cases}1 & \left(b \neq 0,\left(u_{1}, u_{2}\right)=(1,1)\right), \\ 0 & \text { otherwise }\end{cases}
$$


Note that, from the definition,

$$
\frac{1}{\left|\mathcal{B}_{2}\right|\left|\Omega_{Y_{2}}\right|} \sum_{B_{2} \in \mathcal{B}_{2}} \sum_{f_{2} \in \Omega_{Y_{2}}} \phi_{1}\left(I\left(B_{2}\right) f_{2} ; 1,1,1,1\right)=L_{1} C_{Y_{1}}(1,2,3) .
$$

Since

$$
\left(\tilde{b}_{1}, \tilde{b}_{2}, \ldots, \tilde{b}_{L_{2}}\right) \in \mathcal{B}_{2} \longrightarrow\left(\tilde{b}_{i}, \tilde{b}_{i+1}, \ldots, \tilde{b}_{L_{2}}, \tilde{b}_{1}, \ldots, \tilde{b}_{i-1}\right) \in \mathcal{B}_{2}
$$

and

$$
\begin{aligned}
& \sharp\left\{{ }^{\prime} 11 \text { ' in } f_{2} \in \Omega_{Y_{2}}\right\}=\sharp\left\{' 11 \text { ' in } f_{2}^{\prime} \in \Omega_{Y_{2}}\right\}, \\
& \sum_{B_{2} \in \mathcal{B}_{2}} \sum_{i=1}^{L_{2}} \zeta\left(\tilde{b}_{i} ; f_{2}(i), f_{2}(i+1)\right)=\sum_{B_{2} \in \mathcal{B}_{2}} \zeta\left(\tilde{b}_{1} ; 1,1\right)\left(\sum_{i=4}^{P_{1}} k_{i}(i-3)\right)=\left(\begin{array}{c}
L_{2}+k_{2}-2 \\
k_{2}-1
\end{array}\right) L_{2} C_{Y_{2}}(1) .
\end{aligned}
$$

Hence, we have

$$
\begin{aligned}
\mathcal{R}_{3}= & \frac{1}{\left|\mathcal{B}_{2}\right|} \sum_{B_{2} \in \mathcal{B}_{2}}\left\{2\left(3 \hat{k}_{3}+2 k_{2}-j\left(B_{2}\right)\right) \frac{\left(\begin{array}{c}
L_{1}+k_{1}-2 \\
k_{1}-1
\end{array}\right)}{\left(\begin{array}{c}
L_{1}+k_{1}-1 \\
k_{1}
\end{array}\right)}\right. \\
& +\left(L_{1}-4 \hat{k}_{3}-3 k_{2}-j\left(B_{2}\right)-\left(\zeta\left(\tilde{b}_{1} ; 1,1\right)+1\right) \sum_{i=4}^{P_{1}} k_{i}(i-3)\right) \frac{\left(\begin{array}{c}
L_{1}+k_{1}-3 \\
k_{1}-2
\end{array}\right)}{\left(\begin{array}{c}
L_{1}+k_{1}-1 \\
k_{1}
\end{array}\right)} \\
& \left.-\frac{\left(\begin{array}{c}
L_{1}+k_{1}-3 \\
k_{1}-2
\end{array}\right)+\left(\begin{array}{c}
L_{1}+k_{1}-4 \\
k_{1}-2
\end{array}\right)}{\left(\begin{array}{c}
L_{1}+k_{1}-1 \\
k_{1}
\end{array}\right)} \frac{1}{\left|\Omega_{Y_{2}}\right|} \sum_{f_{2} \in \Omega_{Y_{2}}} \phi_{1}\left(I\left(B_{2}\right) f_{2} ; 1,1,1,1\right)\right\} \\
= & \frac{2 k_{1}\left(3 \hat{k}_{3}+2 k_{2}-\widetilde{\mathcal{R}}_{2}\right)}{L_{1}+k_{1}-1} \\
& +\frac{k_{1}\left(k_{1}-1\right)\left\{L_{1}-4 \hat{k}_{3}-3 k_{2}-\widetilde{\mathcal{R}}_{2}-\left(\frac{k_{2}}{L_{2}+k_{2}-1}+1\right) L_{2} C_{Y_{2}}(1)-L_{1} C_{Y_{1}}(1,2,3)\right\}}{\left(L_{1}+k_{1}-1\right)\left(L_{1}+k_{1}-2\right)} \\
& -\frac{k_{1}\left(k_{1}-1\right)\left(L_{1}-1\right) L_{1} C_{Y_{1}}(1,2,3)}{\left(L_{1}+k_{1}-1\right)\left(L_{1}+k_{1}-2\right)\left(L_{1}+k_{1}-3\right)} .
\end{aligned}
$$

From Proposition 2, this equation and $K_{i}^{(j)}$ give (12).

\section{Concluding remarks}

In this article, we showed a recursion formula for the $N$-point functions $C_{Y}(1,2, \ldots, N-1)$. Using the formula, we explicitly calculated the second and the third nearest neighbor correlation functions. In these estimations, we found that $C_{Y}(3)$ is essentially obtained from $C_{Y}(1)$, $C_{Y}(2)$ and $C_{Y}(1,2,3)$. We expect that such recurrence formulae may exist for general $N$-point functions. Obtaining the recurrence formulae and to clarify their relation to correlation functions for quantum integrable systems are problems that will be addressed in the future.

\section{A Evaluation of $\mathcal{R}_{3}$ in $C_{Y}(3)$}

Let $B_{2} \in \mathcal{B}_{2}, f_{2} \in \Omega_{Y_{2}}$ and

$$
\hat{R}\left(B_{2} ; f_{2}\right):=\frac{1}{\left|\mathcal{B}_{1}\right|} \sum_{B_{1} \in \mathcal{B}_{1}}\left(\sharp\left\{' 1101 ', ' 1011 ' \in I\left(B_{1}\right) I\left(B_{2}\right) f_{2}\right\}\right.
$$




$$
\left.-\sharp\left\{\text { ' } 1001 \text { ', ' } 1111 \text { ' } \in I_{1} I\left(B_{2}\right) f_{2} \text { disappear in } I\left(B_{1}\right) I\left(B_{2}\right) f_{2}\right\}\right) .
$$

To evaluate $\hat{R}\left(B_{2} ; f_{2}\right)$ we have to count the balance of the increment and the decrement of the $1 * * 1$ patterns by 10 -insertion. There are two types of such $1 * * 1$ patterns:

(A) One of the $1 \mathrm{~s}$ at the ends of a $1 * * 1$ pattern originally belong to $I_{1} I\left(B_{2}\right) f_{2}$;

(B) Both of the $1 \mathrm{~s}$ at the ends come from 10-insertion $I_{2}\left(B_{1}\right)$.

In each case, the variation is listed as follows.

(A) Let $f_{1}=I\left(B_{2}\right) f_{2}$. When a block $\underbrace{10 \underline{10} \cdots \underline{10}}_{b_{n} \neq 0}$ is inserted between $f_{1}(n)$ and $f_{1}(n+1)$, the number of $1 * * 1$ patterns of type $(\mathrm{A})$ is given as:

(1) $f_{1}(n) f_{1}(n+1)=\mathbf{0 0}$ :

(a) $0000 \longrightarrow 00 \underline{10} \underline{10} \cdots \underline{1000} \Rightarrow \pm 0(+0,-0)$,

(b) $110000 \longrightarrow 1100 \underline{10} \underline{10} \cdots \underline{1000} \Rightarrow+1(+1,-0)$,

(c) $0001 \longrightarrow 00 \underline{10} \underline{10} \cdots \underline{1001} \Rightarrow+1(+1,-0)$,

(d) 1 10001 $\rightarrow 10 \underline{10} \underline{10} \underline{10} \cdots \underline{1001} \Rightarrow+2(+2,-0)$;

(2) $f_{1}(n) f_{1}(n+1)=\mathbf{1 0}$ :

(a) $11000 \longrightarrow 11010 \underline{10} \cdots \underline{1000} \Rightarrow+1(+1,-0)$,

(b) $\mathbf{1 1 0} 01 \longrightarrow \mathbf{1} 10 \underline{10} \underline{10} \cdots \underline{10} 01 \Rightarrow+1(+2,-1)$;

(3) $f_{1}(n) f_{1}(n+1)=\mathbf{0 1}$ :

(a) $0011 \longrightarrow 00 \underline{10} \underline{10} \cdots \underline{1011} \Rightarrow+1(+1,-0)$,

(b) 1 10011 $\rightarrow 10 \underline{10} \underline{10} \underline{10} \cdots \underline{1011} \Rightarrow+1(+2,-1)$;

(4) $f_{1}(n) f_{1}(n+1)=\mathbf{1 1}$ :

(a) $011 \underline{100} \rightarrow 01 \underline{10} \underline{10} \cdots \underline{101} 100 \Rightarrow+2(+2,-0)$,

(b) $0111[100 \longrightarrow 011 \underline{10} \underline{10} \cdots \underline{101} 100 \Rightarrow+1(+2,-1)$,

$\left(\mathrm{b}^{\prime}\right) 1111 \underline{100} \longrightarrow 111 \underline{10} \underline{10} \cdots \underline{101} 100 \Rightarrow+1(+3,-2)$,

(c) $0111 \rightarrow 01 \underline{10} \underline{10} \cdots \underline{1011} \Rightarrow+1(+2,-1)$,

(d) $011111 \rightarrow 011 \underline{10} \underline{10} \cdots \underline{10111} \Rightarrow \pm 0(+2,-2)$,

$\left(\mathrm{d}^{\prime}\right) 111111 \longrightarrow 111 \underline{10} \underline{10} \cdots \underline{10} 111 \Rightarrow \pm 0(+3,-3)$,

where $+1(+1,-0)$ etc. denote the numbers of the total (created, disappeared) $1 * * 1$ patterns.

Note that total number does not depend on the length of the blocks and that the variation of $1 * * 1$ patterns is determined for the four elements $f_{1}(n-2) f_{1}(n-1) f_{1}(n) f_{1}(n+1)$. Hence, for $b_{n} \neq 0$, the contribution from a boundary and a block is summarized as

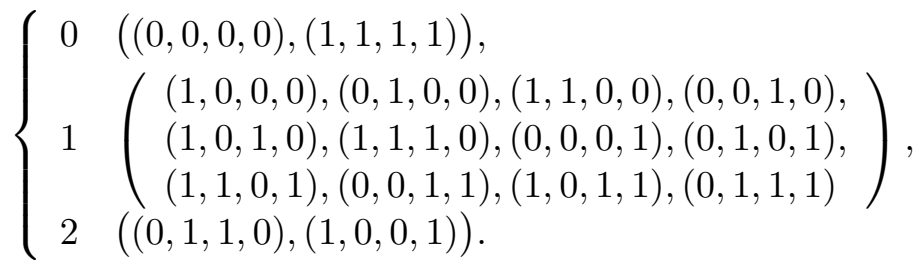

For example, there is no contribution for $\left(f_{1}(n-1), f_{1}(n), f_{1}(n+1), f_{1}(n+2)\right)=(0,0,0,0)$, $(1,1,1,1)$, and +2 for $\left(f_{1}(n-1), f_{1}(n), f_{1}(n+1), f_{1}(n+2)\right)=(0,1,1,0),(1,0,0,1)$.

(B) When blocks are inserted between $f_{1}(n-1)$ and $f_{1}(n)$ and between $f_{1}(n)$ and $f_{1}(n+1)$ $\left(b_{n-1} \neq 0, b_{n} \neq 0\right)$, the total number of $1 * * 1$ patterns generated between two blocks is given as: 
(1) $f_{1}(n-1) f_{1}(n) f_{1}(n+1)=\mathbf{0 0 0}$ :

$\longrightarrow 0 \underline{10} \cdots \underline{10} 0 \underline{10} \underline{10} \cdots \underline{100} \Rightarrow(+1, \pm 0)$,

(2) $f_{1}(n-1) f_{1}(n) f_{1}(n+1)=\mathbf{0 0 1}$ :

$\longrightarrow 0 \underline{10} \cdots \underline{100} \underline{10} \underline{10} \cdots \underline{10} 1 \Rightarrow(+1,-1)$,

(3) $f_{1}(n-1) f_{1}(n) f_{1}(n+1)=\mathbf{0 1 0}$ :

$\longrightarrow 0 \underline{10} \cdots \underline{101} 1010 \underline{10} \cdots \underline{100} \Rightarrow( \pm 0, \pm 0)$,

(4) $f_{1}(n-1) f_{1}(n) f_{1}(n+1)=\mathbf{0 1 1}$ :

$\longrightarrow 0 \underline{10} \cdots \underline{10} 1 \underline{10} \underline{10} \cdots \underline{10} 1 \Rightarrow(+1,-1)$,

(5) $f_{1}(n-1) f_{1}(n) f_{1}(n+1)=\mathbf{1 0 0}$ :

$\longrightarrow 1 \underline{1010} \cdots \underline{100} \underline{10} \underline{10} \cdots \underline{100} \Rightarrow(+1,-1)$,

(6) $f_{1}(n-1) f_{1}(n) f_{1}(n+1)=\mathbf{1 0 1}$ :

$\longrightarrow 110 \underline{10} \cdots \underline{100} \underline{10} \underline{10} \cdots \underline{10} 1 \Rightarrow(+1,-2)$,

(7) $f_{1}(n-1) f_{1}(n) f_{1}(n+1)=\mathbf{1 1 0}$ :

$\longrightarrow 1 \underline{10} \cdots \underline{10} 1010 \underline{10} \cdots \underline{100} \Rightarrow( \pm 0, \pm 0)$,

(8) $f_{1}(n-1) f_{1}(n) f_{1}(n+1)=\mathbf{1 1 1}$ :

(a) $\longrightarrow 01 \underline{10} \cdots \underline{101} 1 \underline{10} \underline{10} \cdots \underline{101} \Rightarrow(+1,-1)$,

(b) $\longrightarrow 11 \underline{10} \cdots \underline{101} \underline{10} \underline{10} \cdots \underline{101} \Rightarrow(+1,-2)$;

Here $(+1, \pm 0)$ etc. denote the number of $1 * * 1$ patterns which (arise between blocks, are eliminated by the 10-insertion of blocks).

When a block is inserted between $f_{1}(n-2)$ and $f_{1}(n-1)$ and another block is inserted between $f_{1}(n)$ and $f_{1}(n+1)$;

(9) $f_{1}(n-1) f_{1}(n) f_{1}(n+1)=\mathbf{1 1 1}$ :

(a) $\longrightarrow 0 \underline{10} \cdots \underline{1011} \underline{10} \underline{10} \cdots \underline{101} \Rightarrow( \pm 0, \pm 0)$,

(b) $\longrightarrow 1 \underline{10} \cdots \underline{1011} \underline{10} \underline{10} \cdots \underline{101} \Rightarrow( \pm 0,-1)$.

From these list, we find that the pattern $f_{1}(n-1) f_{1}(n) f_{1}(n+1) f_{1}(n+2)$ determines the variation locally. For $b_{n} b_{n+1} \neq 0$,

$$
\left\{\begin{aligned}
1 & ((1,0,0,0),(0,0,0,0)), \\
-1 & \left(\begin{array}{l}
(1,1,0,1),(0,1,0,1), \\
(1,1,1,1)
\end{array}\right) \\
0 & \text { otherwise }
\end{aligned}\right.
$$

and for $b_{n-1} \neq 0, b_{n}=0, b_{n+1} \neq 0$,

$$
\left\{\begin{aligned}
-1 & (1,1,1,1) \\
0 & \text { otherwise. }
\end{aligned}\right.
$$

Therefore, using the functions $\Phi_{1}, \Phi_{2}$ and $\Phi_{3}(7)-(9)$ in Proposition 2, we obtain

$$
\begin{aligned}
\hat{R}\left(B_{2} ; f_{2}\right)= & \frac{1}{\left|\mathcal{B}_{1}\right|} \sum_{B_{1} \in \mathcal{B}_{1}} \sum_{i=1}^{L_{1}} \\
& \left(\Phi_{1}\left(b_{i} ; I\left(B_{2}\right) f_{2}(i-1), I\left(B_{2}\right) f_{2}(i), I\left(B_{2}\right) f_{2}(i+1), I\left(B_{2}\right) f_{2}(i+2)\right)\right. \\
& +\Phi_{2}\left(b_{i}, b_{i+1} ; I\left(B_{2}\right) f_{2}(i-1), I\left(B_{2}\right) f_{2}(i), I\left(B_{2}\right) f_{2}(i+1), I\left(B_{2}\right) f_{2}(i+2)\right) \\
& \left.+\Phi_{3}\left(b_{i-1}, b_{i+1} ; I\left(B_{2}\right) f_{2}(i-1), I\left(B_{2}\right) f_{2}(i), I\left(B_{2}\right) f_{2}(i+1), I\left(B_{2}\right) f_{2}(i+2)\right)\right) .
\end{aligned}
$$




\section{B Derivation of equations (13), (14) and (15)}

From (17) and Proposition 2, we find

$$
\hat{R}\left(B_{2} ; f_{2}\right)=\frac{1}{\left|\mathcal{B}_{1}\right|} \sum_{B_{1} \in \mathcal{B}_{1}} \sum_{\boldsymbol{b} \in\left(\mathbb{F}_{2}\right)^{4}} \phi_{1}\left(I\left(B_{2}\right) f_{2} ; \boldsymbol{b}\right)\left(\Phi_{1}\left(b_{1} ; \boldsymbol{b}\right)+\Phi_{2}\left(b_{1}, b_{2} ; \boldsymbol{b}\right)+\Phi_{3}\left(b_{1}, b_{3} ; \boldsymbol{b}\right)\right) \text {. }
$$

Let $f_{1}:=I\left(B_{2}\right) f_{2}$. First we consider the term:

$$
\begin{aligned}
\hat{R}_{A}\left(B_{2} ; f_{2}\right) & :=\frac{1}{\left|\mathcal{B}_{1}\right|} \sum_{B_{1} \in \mathcal{B}_{1}} \sum_{\boldsymbol{b} \in\left(\mathbb{F}_{2}\right)^{4}} \phi_{1}\left(I\left(B_{2}\right) f_{2} ; \boldsymbol{b}\right) \Phi_{1}\left(b_{1} ; \boldsymbol{b}\right) \\
& =\frac{1}{\left|\mathcal{B}_{1}\right|} \sum_{B_{1} \in \mathcal{B}_{1}} \sum_{n=1}^{L_{1}} \Phi_{1}\left(b_{1} ; f_{1}(n-1), f_{1}(n), f_{1}(n+1), f_{1}(n+2)\right) .
\end{aligned}
$$

For $b \neq 0$, we observe $\Phi_{1}(b ; \mathbf{b})=\sharp\left\{{ }^{\prime} 10^{\prime},{ }^{\prime} 01 ' \in \mathbf{b}\right\}-\sharp\left\{{ }^{\prime} 101{ }^{\prime},{ }^{\prime} 010^{\prime} \in \mathbf{b}\right\}$. There are $\hat{k}_{2}$ ' 10 's, the same number of ' 01 's and $j\left(B_{2}\right)+\sharp\{$ ' 10 ' inserted between consecutive 1's $\}$ ' 101 's in $f_{1}$. Since the number of ' 010 's is equal to $\sharp\left\{{ }^{\prime} \underline{10}\right.$ ' $\}-\sharp\left\{\underline{10}^{\prime}\right.$ ' inserted between consecutive 1 's $\}$, we find

$$
\hat{R}_{A}\left(B_{2} ; f_{2}\right)=6 \hat{k}_{2}-2 j\left(B_{2}\right)-2 k_{2} \text {. }
$$

The rest terms can be evaluated by counting the numbers of 3 -tuples in $f_{1}$.

- the number of $\left(f_{1}(n-1), f_{1}(n), f_{1}(n+1)\right)=(0,0,1),(1,0,0)$ is

$$
2 \hat{k}_{2}-\sharp\left\{n \in\left[L_{2}\right] \mid\left(f_{1}(n-1), f_{1}(n), f_{1}(n+1)\right)=(1,0,1)\right\} ;
$$

- the number of $\left(f_{1}(n-1), f_{1}(n), f_{1}(n+1)\right)=(0,1,0)$ is

$$
k_{2}-\sum_{i=1}^{L_{2}} \zeta\left(\tilde{b}_{i} ; f_{2}(i), f_{2}(i+1)\right)
$$

where $\zeta\left(b ; u_{1}, u_{2}\right)$ is defined by $(16)$;

- the number of $\left(f_{1}(n-1), f_{1}(n), f_{1}(n+1)\right)=(0,1,1),(1,1,0)$ is

$$
2 \hat{k}_{3}+2 \sum_{i=1}^{L_{2}} \zeta\left(\tilde{b}_{i} ; f_{2}(i), f_{2}(i+1)\right)
$$

- the number of $\left(f_{1}(n-1), f_{1}(n), f_{1}(n+1)\right)=(1,0,1),(1,1,1)$ is

$$
\sum_{i=4}^{P_{1}} k_{i}(i-3)+j\left(B_{2}\right)
$$

Hence we have

$$
\begin{aligned}
& \sharp\left\{{ }^{\prime} 000^{\prime} \in I\left(B_{2}\right) f_{2}\right\}-\sharp\left\{{ }^{\prime} 101 ' \in I\left(B_{2}\right) f_{2}\right\} \\
& \quad=L_{1}-4 \hat{k}_{3}-3 k_{2}-j\left(B_{2}\right)-\sum_{i=1}^{L_{2}} \zeta\left(\tilde{b}_{i} ; f_{2}(i), f_{2}(i+1)\right)-\sum_{i=4}^{P_{1}} k_{i}(i-3) .
\end{aligned}
$$

Therefore

$$
\begin{aligned}
& \phi_{1}\left(I\left(B_{2}\right) f_{2} ; 1,0,0,0\right)+\phi_{1}\left(I\left(B_{2}\right) f_{2} ; 0,0,0,0\right)-\phi_{1}\left(I\left(B_{2}\right) f_{2} ; 1,1,0,1\right) \\
& \quad-\phi_{1}\left(I\left(B_{2}\right) f_{2} ; 0,1,0,1\right)=\sharp\left\{{ }^{\prime} 000{ }^{\prime} \in I\left(B_{2}\right) f_{2}\right\}-\sharp\left\{{ }^{\prime} 1011^{\prime} \in I\left(B_{2}\right) f_{2}\right\} \\
& \quad=L_{1}-4 \hat{k}_{3}-3 k_{2}-j\left(B_{2}\right)-\sum_{i=1}^{L_{2}} \zeta\left(\tilde{b}_{i} ; f_{2}(i), f_{2}(i+1)\right)-\sum_{i=4}^{P_{1}} k_{i}(i-3) .
\end{aligned}
$$

From (18) and (19), $\hat{R}\left(B_{2} ; f_{2}\right)$ can be obtained as shown in the proof of Theorem 3. 


\section{Acknowledgements}

The authors wish to thank Professor Atsushi Nagai for useful comments.

\section{References}

[1] Takahashi D., Satsuma J., A soliton cellular automaton, J. Phys. Soc. Japan 59 (1990), 3514-3519.

[2] Yura F., Tokihiro T., On a periodic soliton cellular automaton, J. Phys. A: Math. Gen. 35 (2002), 3787-3801, nlin.SI/0112041.

[3] Fukuda K., Okado M., Yamada Y., Energy functions in box ball systems, Internat. J. Modern Phys. A 15 (2000), 1379-1392, math.QA/9908116.

[4] Hatayama G., Hikami K., Inoue R., Kuniba A., Takagi T., Tokihiro T., The $A_{M}^{(1)}$ automata related to crystals of symmetric tensors, J. Math. Phys. 42 (2001), 274-308, math.QA/9912209.

[5] Mada J., Tokihiro T., Correlation functions for a periodic box-ball system, J. Phys. A: Math. Theor. 43 (2010), 135205, 15 pages, arXiv:0911.3953.

[6] Jimbo M., Miwa T., Quantum KZ equation with $|q|=1$ and correlation functions of the $X X Z$ model in the gapless regime, J. Phys. A: Math. Gen. 29 (1996), 2923-2958.

[7] Mada J., Idzumi M., Tokihiro T., The exact correspondence between conserved quantities of a periodic box-ball system and string solutions of the Bethe ansatz equations, J. Math. Phys. 47 (2006), 053507, 18 pages.

[8] Torii M., Takahashi D., Satsuma J., Combinatorial representation of invariants of a soliton cellular automaton, Phys. D 92 (1990), 209-220. 